\title{
The Delivery of Web Mining in Healthcare System on Cloud Computing
}

\author{
Nidhi Solanki, Aman Duraja \\ PDM College of Engineering \\ (Affiliated to M D University, Rohtak, haryana)
}

\begin{abstract}
In Current economic trends The Healthcare organizations are seeking ways to improve their workflows and information management to discover knowledge for understanding the cause of any disease and its treatment, more accurate control of medicines and supplies, faster coding and billing, and efficient patient processes that support optimal care more flexible. The construction, delivery \& management of software services through Cloud Computing have been changed radically. Cloud computing provides computation, software, data access, and storage. These services are great innovation in the field of computing. So now it has become indispensable to the healthcare organizations to adapt new technology and new ideas to develop new information that meet the need of current business requirements. Internet has played a vital \& crucial role in the business environment in current scenario, the term "Cloud computing" is quite important because of their versatility as they serve for enterprises and individuals around the globe. The Cloud Computing deliver many business advantages like computation, data access, storage, Zero install, Reduce business costs, Centralized data, Quick and easy updating and make all these available $24 x 7$ hour from anywhere any time through any internet compatible devices. However there are certain aspects related to the issues which are needed to get attention and handle cautiously.

In our present study various advantages while delivering healthcare services on cloud computing like unlimited storage, Flexible, Backup \& Recovery, Automatic software Integration, Quick Deployment, Easy Access to Information, Cost Efficient are critically analyzed, synthesized. The outcome of the study would be of a great significance to deal with the issues for future enhancement.

Keyword: Storage, Data Integration, Backup \& Recovery, Security and Privacy, Quick Deployment, Flexibility, Decrease Cost.
\end{abstract}

Submitted date 17 .June $2013 \quad$ Accepted Date: 22 .June 2013

\section{A. About Healthcare}

\section{Introduction}

The health care industry or medical industry is a sector within the economic system that provides goods and services to treat patients with curative, preventive, rehabilitative, and palliative care. The modern health care sector is divided into many sub-sectors, and depends on interdisciplinary teams of trained professionals and paraprofessionals to meet health needs of individuals and populations. The health care industry is one of the world's largest and fastest-growing industries. Consuming over 10 percent of gross domestic product (GDP) of most developed nations, health care can form an enormous part of a country's economy. Healthcare organizations of all sizes face a critical need to manage and integrate clinical, financial and operational information. Our Hospital Information Management (HIM) offers a complete, modular and agile hospital information system that is flexible, affordable and intuitive. The solution has been developed from the ground up with a key focus of meeting the needs of healthcare professionals while minimizing the costs and resources required running global healthcare organizations.

\section{B. About Web Mining}

The web is a means for accessing a vast variety of information stored in various parts of the world. Information is mostly in the form of unstructured data i.e. Web is the single largest data source in the world. Due to heterogeneity and lack of structure of web data, mining is a challenging task. Web mining helps companies or healthcare organization or hospital to collect and analyze data. Web Mining can help optimize system performance and derive meaningful information from disparate data sources.

Suppose before purchasing the healthcare product or medicine a hospital can compare the price of various suppliers companies so that the hospital can purchase economic product. If the hospital purchase economic product then the hospital provide economical treatment. 


\section{C. $\quad$ About Cloud Computing}

The construction, delivery \& management of software services through Cloud Computing have been changed radically. Cloud computing provides computation, software, data access, and storage services over the Internet. These services are broadly divided into three categories: Infrastructure-as-a-Service (IaaS), Platformas-a-Service (PaaS) and Software-as-a-Service (SaaS).

SaaS: - Software as a Service (SaaS) is a software distribution model in which applications are hosted by a vendor or service provider and made available to customers over a network, typically the Internet.

PaaS: - Platform as a Service (PaaS) is a way to rent hardware, operating systems, storage and network capacity over the Internet. The service delivery model allows the customer to rent virtualized servers and associated services for running existing applications or developing and testing new ones.

IaaS: - Infrastructure as a Service is a provision model in which an organization outsources the equipment used to support operations, including storage, hardware, servers and networking components. The service provider owns the equipment and is responsible for housing, running and maintaining it. The client typically pays on a per-use basis.

\section{Proposed Model}

In this research proposed technique help in much efficient way to identify the healthcare related activities like product price comparison, facilities comparison, patient history, patient treatment history, fast admission, fast billing, Online appointments, Doctors information, Disease Information and Online support from anywhere any time.

These all facilities are delivered by a cloud based server and accordingly it becomes easy for end users, patients, organizations, researchers \& practitioners to access or to extract the required data or information through the Internet compatible appliances like PDAs, laptops, desktop computers and other such devices access information from the web server.

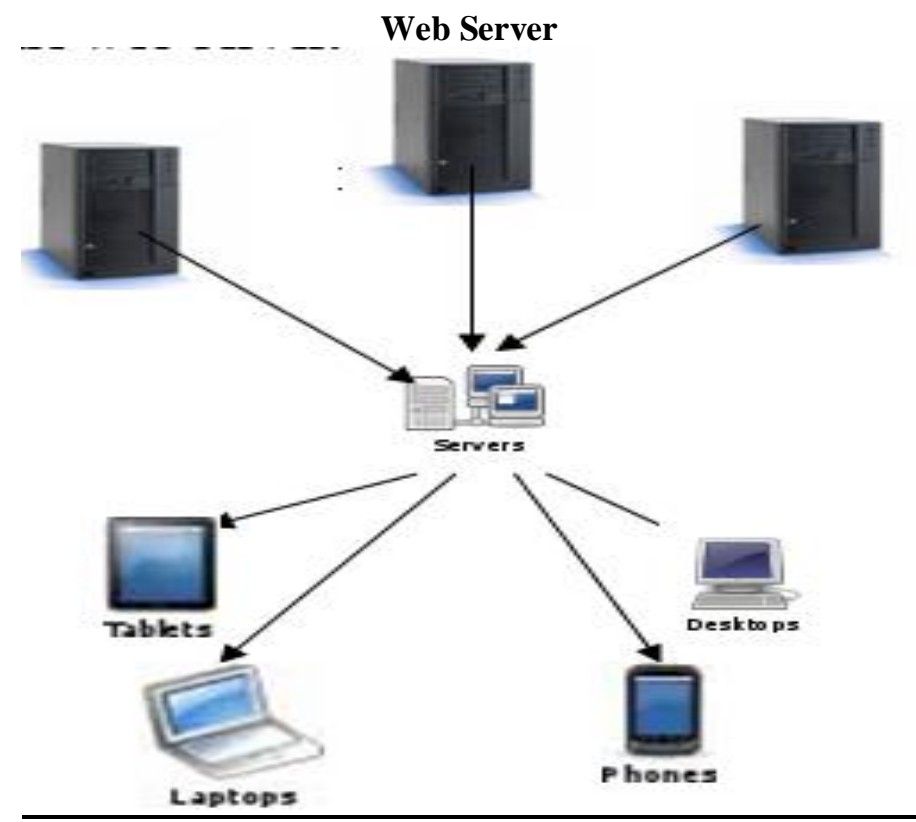

Fig 1: To deliver cloud based web mining services in healthcare

It is a new paradigm in which computing is migrating from personal computers to large, centrally managed datacenters. It is the cloud based computing where virtual shared server provides computation, software, data access, and storage services. End-user or customer doesn't have the knowledge of the physical location and configuration of the system that delivers the services. It is economic, Faster, simpler and cheaper to use. Applications can be accessed from anywhere anytime.

\section{Characteristics of this approach}

1. Device and location independence: the Internet, users can connect from anywhere any time through any devices (PC, smart phone) using a web browser.

2. Security: improve due to centralization of data, increased security-focused resources, etc., but concerns can persist about loss of control over certain sensitive data. Security is often as good as or better than under traditional systems, in part because providers are able to devote resources to solving security issues that many 
customers cannot afford. However, the complexity of security is greatly increased when data is distributed over a wider area or greater number of devices and in multi-tenant systems that are being shared by unrelated users.

3. Increased Scalability

a. Increase Capacity 24 hours

b. Capacity on demand

4. Increased Speed

a. $\quad 24$ hour provisioning

b. Online self service

5. Cost Efficient: Cloud computing is probably the most cost efficient method to use, maintain and upgrade. Traditional desktop software costs companies a lot in terms of finance. Adding up the licensing fees for multiple users can prove to be very expensive for the establishment concerned. The cloud, on the other hand, is available at much cheaper rates and hence, can significantly lower the company's IT expenses.

6. Almost Unlimited Storage: Storing information in the cloud gives you almost unlimited storage capacity. Hence, you no more need to worry about running out of storage space or increasing your current storage space availability.

7. Backup and Recovery: Since all your data is stored in the cloud, backing it up and restoring the same is relatively much easier than storing the same on a physical device.

8. Automatic Software Integration: In the cloud, software integration is usually something that occurs automatically. This means that you do not need to take additional efforts to customize and integrate your applications as per your preferences.

This research mainly concentrates on providing better approach for delivering web data in healthcare more accurately. Experimental results suggest the significance of the proposed approach. Step 1: $1^{\text {st }}$ the hospital extract data from given websites.

Step 2: Then cleaning and integration of the extracted data and the transform it into meaningful information and storage it into database.

Step 3: Then the users can use it from anywhere any time through any internet compatible appliances.

The procedure we follow is given below graphically.

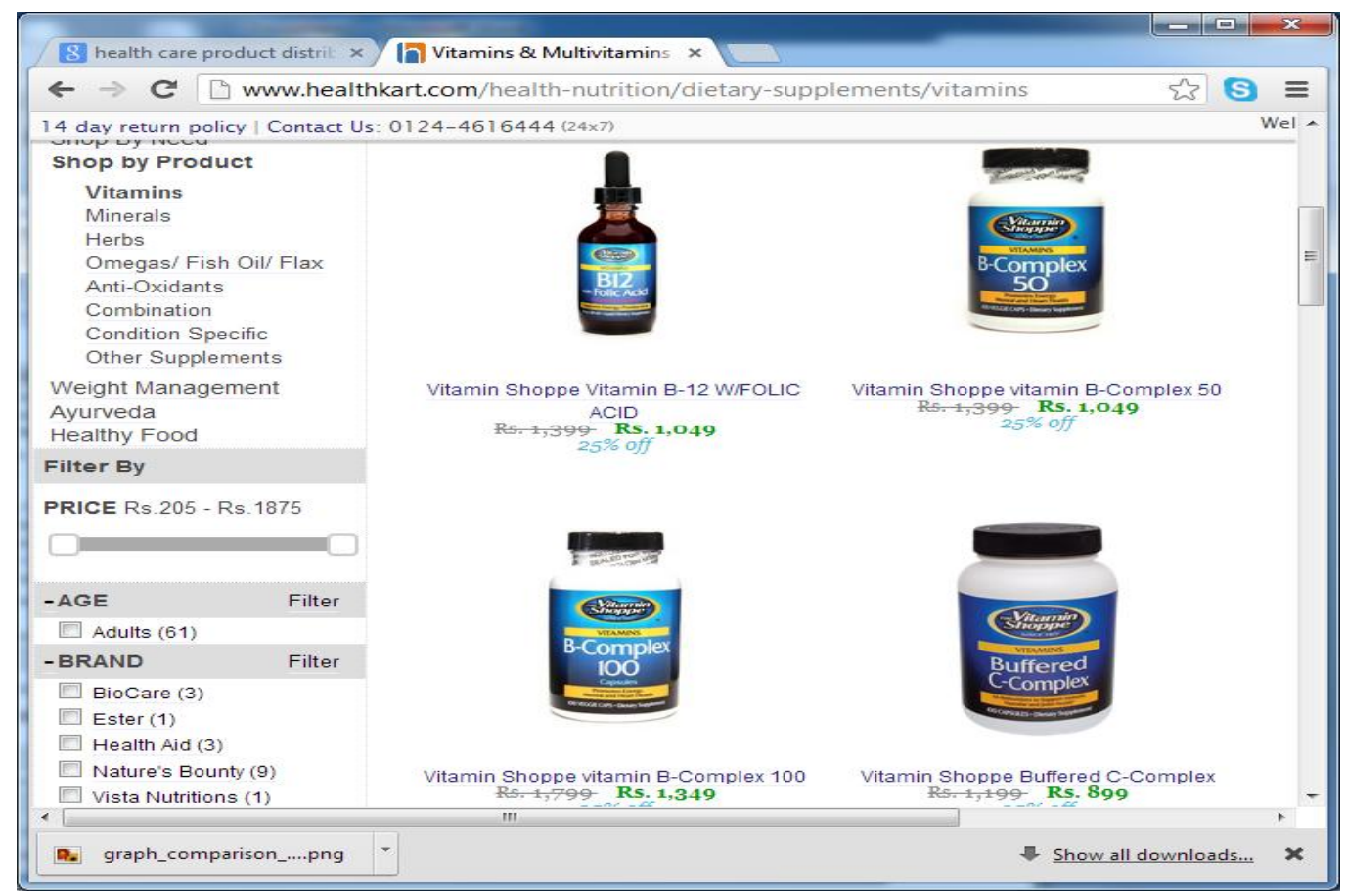

Fig 1: First website Screen shot 


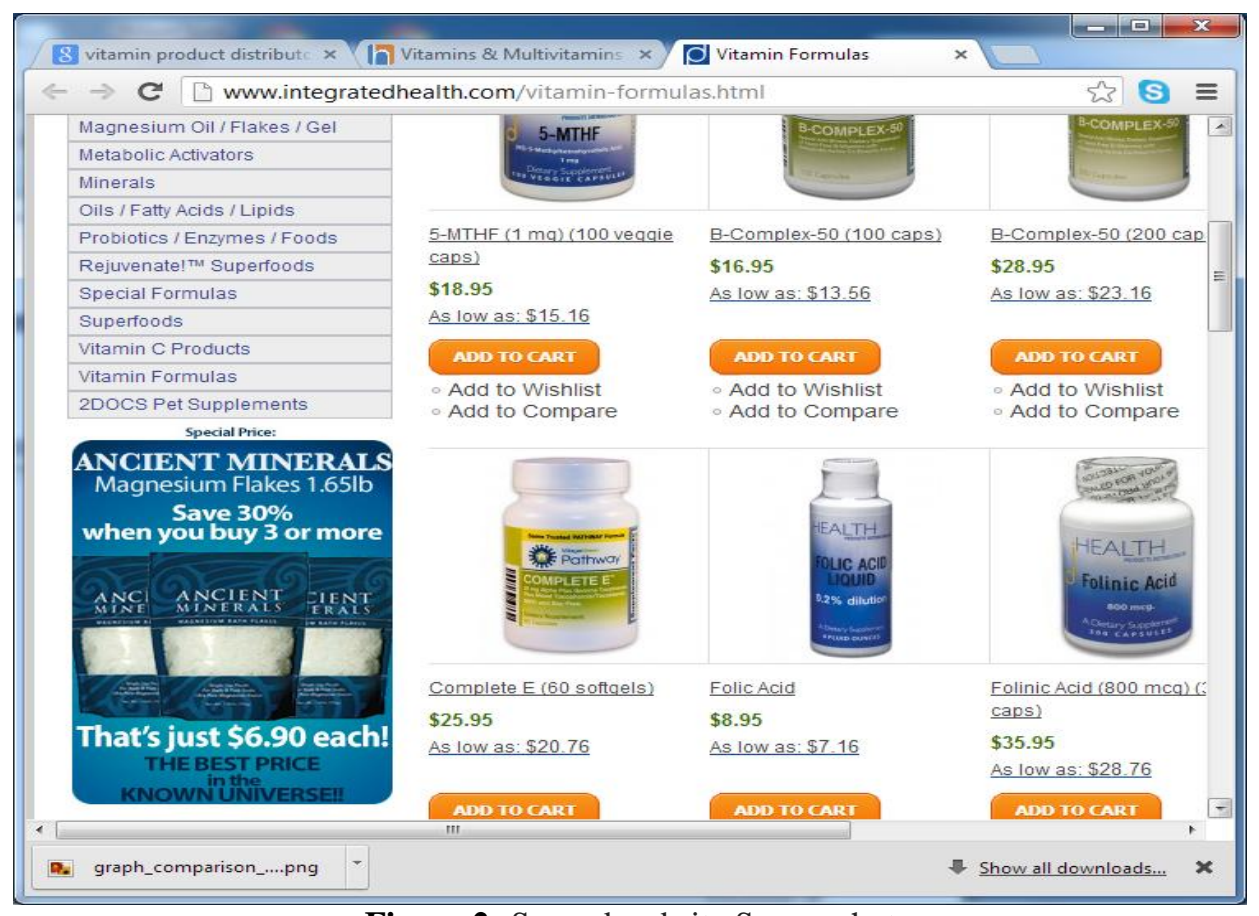

Figure 2: Second website Screen shot

Above figures are the online suppliers of healthcare product. Before purchasing the healthcare product hospital can compare the price using web mining module. The module can extract data from source and compare the all health care product price graphically using column chart. After comparing price of product the hospital can decide from where to purchase healthcare product, so that the operational or treatment cost is minimized.The output is given as follows.

\section{The output is like this}

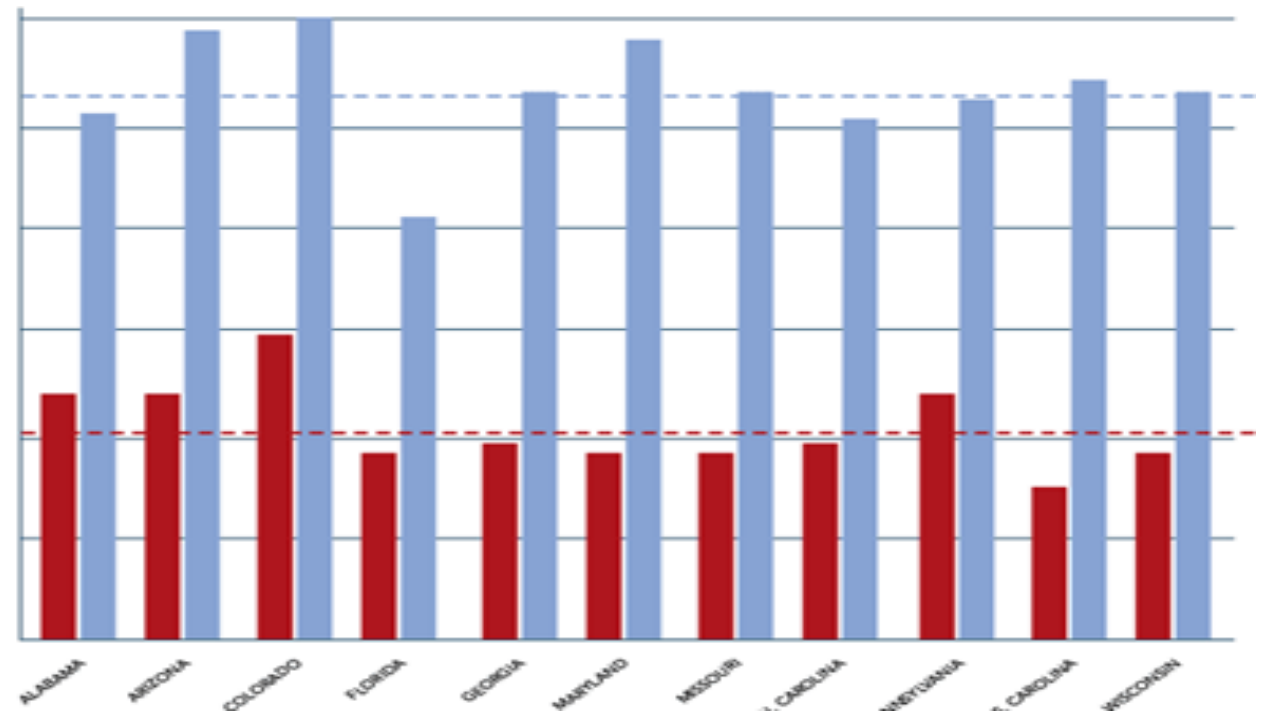

IV. Conclusion

Like everything else, cloud computing too has its pros and cons. While the technology can prove to be a great asset to your company, it could also cause harm if not understood and used properly. Two decades ago such approximations were impossible simply because neither the technology nor the computer capacity were available. Today's landscape is quite different. Many applications are better served. The Experimental results suggest the significance of the proposed approach. 


\section{References}

[1] Dr. M. Giri and Dr. Akash Kumar, “An Efficient Web Content Mining using elevance Analysis Approach”, International Journal of Multidisciplinary Research in Advanced Engineering, pages. 201-210, 2012

[2] Dr. M. Giri and Dr. Akash Kumar, “An Efficient Web Content Mining using Divide and Conquer Approach”, International journal of Computational Intelligence Research, pages. 201-210, 2012.

[3] Dr. M. Giri and Dr. Akash Kumar, "An Efficient Web Content Mining using Multi Threading Approach", International Journal of Systems, Algorithms and Applications, pages. 1-4, 2012

[4] Yan LI, Boqin FENG and Qinjiao MAO, "Research on Path Completion Technique In Web Usage Mining", IEEE International Symposium On Computer Science and Computational Technology, pp. 554-559, 2008.

[5] Batista, G. \& Monard, M., 2003, 'An analysis of four missing data treatment methods for supervised learning', Applied Artificial Intelligence, vol. 17, no. 5-6, pp. 519-533.

[6] C. Romero, S. Ventura "Educational data Mining: A Survey from 1995 to 2005", Expert Systems with Applications (33), pp. 135146, 2007

[7] R.Cooley, B. Mobasher, and J. Srivastava. "Web mining: Information and Pattern Discovery on the World Wide Web." In Proceedings of Ninth IEEE International Conference on Tools with Artificial Intelligence” (ICTAI'97), November 1997.

\section{About Author:}

Ms. Nidhi Solanki is the student of M. tech at PDM College of Engineering. She has received B.Tech degree from BMIET Sonipat Haryana. She has presented various research papers. Her current area of research is Web Mining in HealthCare Industries.

Mr. Aman Dureja is assistant professor at PDM College of Engineering. He has received B.Tech degree from BITS Bhiwani, Haryana in 2007 and M.tech from PDM College of Engineering in 2010. He has presented 15 national and international research papers. 